\title{
A Study on the Application of the Behavior Setting Concept in User Behavior Simulation for Atypical Architectural Design
}

\author{
Su Jin Kim ${ }^{1}$, Hong Jung Kim², Yun Gil Lee ${ }^{3}$ \\ ${ }^{1}$ Student, Department of Architecture, Hoseo University, Korea, tnwls1797@ hanmail.net \\ ${ }^{2}$ Student, Department of Architecture, Hoseo University, Korea, kyong5666@naver.com \\ ${ }^{3}$ Professor, Department of Architecture, Hoseo University, Korea, yglee@ hoseo.edu
}

Corresponding author: Yun Gil Lee

\begin{abstract}
Architects generally predict user behavior in a designed space based on laws or on their own experiences with the architectural design process. However, it is not easy to predict user behavior in atypical-type buildings because there are many cases therein that architects have not experienced. For this reason, human behavior simulation technology is essential in atypical architectural designs. In order to carry out a successful user behavior simulation, it is necessary to reproduce the social interactions occurring in an atypical space. This is because a more natural representation of social phenomena can show architects the potential performance of a more casual architectural space. This study aimed to develop a technology for reproducing behavioral settings for advanced human behavior simulations. In a previous study, social interactions in atypical spaces were classified into several categories based on psychological theories, one of which is behavioral setting. The purpose of the present study was to develop a technology that can be used to further enhance user interaction in an atypical space. In detail, the behavioral setting phenomenon, one of the user interactions, is introduced into the simulation technology. To this end, research was conducted to be able to add a behavioral setting function to ActoViz, which was developed for user interaction simulation in an unstructured space. Through the development of some prototypes, it was confirmed that the reproduction of the behavior setting concept can be expressed in a more advanced user simulation.
\end{abstract}

Keywords: Atypical Architecture, Human Behavior Simulation, Behavioral Setting, Social Interaction, Architectural Design Process

\section{Introduction}

Architectural space regulates human behavior and serves as the basis for social phenomena. Buildings act as containers of human behavior, so different types of buildings can create different types of social phenomena[1]. In this context, atypical architectural spaces can lead to unpredictable user behaviors because these are not the typical forms that users have experienced previously. Architects generally predict user behavior in a designed space based on laws or on their own experiences in the architectural design process. However, it is not easy to predict user behavior in an atypical building where the architect does not have sufficient previous experience of the space. For this reason, human behavior simulation technology is essential in atypical architectural design. In the previous research, the behaviors of users in atypical architectural spaces have been predicted. In other words, an agent technology corresponding to the physical properties of an atypical space has been developed[2]. In order to carry out a more successful user behavior simulation, it is necessary to

Received: May 09, 2021; $1^{\text {st }}$ Review Result: June 25, 2021; $2^{\text {nd }}$ Review Result: August 16, 2021

Accepted: September 30, 2021 
reproduce the social interactions in an atypical space. This is because a more natural representation of social phenomena can reveal to architects the potential performance of an architectural space more closely[3].

This study is carried out as part of a technology development to advance human behavior simulation in atypical spaces. To wit, it is intended for the development of technology that reproduces the social interactions that take place in an atypical architectural space. In a previous study, social interactions in an atypical space have been classified into several types based on psychological theories, one of which is behavioral setting[4]. Existing human behavior simulation technology has limitations that do not consider social interaction such as behavioral setting[3]. This study describes the theoretical background of the technical aspects of behavior setting and presents the strategies and results of their development. In addition, a simulation is performed by loading the previously developed behavior setting technology into ActoViz, which has been developed for user interaction simulation[2].

\section{Architectural Context and Human Behavior}

Designing a space means preparing an environment for user actions. For this undertaking, it is necessary to consider user actions based on context (Wagner 1979). From this perspective, space design can be perceived as a place-making process. Designing a building means that the users' actions or interactions that are developed through physical shapes can be the final result. User interaction is a process that promotes desirable behavior based on social and cultural consensus and takes place in the space designed by architects. In other words, the shape of an architectural space is a major cause of social phenomena, and one of the main factors determining the quality of an architectural space is an appropriate social phenomenon[5].

According to previous research, there are various interpretations of the characteristics of architectural spaces that cause social phenomena. The theory related to the so-called space syntax focuses on the syntactic characteristics of space. Syntactical characteristic refers to the relationship between space and space; it is based on the fact that although the shapes of spaces are similar, the connections between the spaces of each internal space differ. Regardless of the apparent similarity in the shapes of spaces, the user who lives in the space uses the space in a different way and perceives it as a different space. Thus, the syntactic characteristics of this space become important macroscopic factors that induce social phenomena[6]. In addition, Gibson (1977)[7] introduced the concept of affordance and presented the potential for the behavioral induction of physical shapes. That is, specific forms have the potential to induce specific behaviors and are linked to social behavioral characteristics. This concept is widely used in research, such as in human-computer interaction (HCI), as a consideration in designing for users[7]. This is also explained by the semantic characteristics of an architectural space, which refer to the tendency of a space to induce specific actions required of users who use the space or physical environment through the meaning of the space or physical environment. Norman (1999)[8] explained affordance a little more broadly: affordances are action possibilities resulting from physical information, as well as goals, values, beliefs, and past experiences, of the actor. Thus, affordances are perceived as action possibilities[8]. This means that user behavior is determined by combining not only physical space information but also social and psychological information.

After all, the architectural context can be interpreted as the interaction of users through the physical space rather than the physical space itself. Since this is a kind of phenomenon, it is latent and invisible until the user's action occurs. However, it is a self-evident fact that the possibility of such user behavior determines the quality of the building. 


\section{Human Behavior Simulation for Atypical Architectural Design}

Digital tools related to architectural design are being actively used to support designers' productivity and to present possibilities beyond their experience and knowledge. Recently, as the importance of the performance of buildings has become more recognized, various technologies for predicting the safety, economic feasibility, and eco-friendliness of buildings have been developed and utilized.

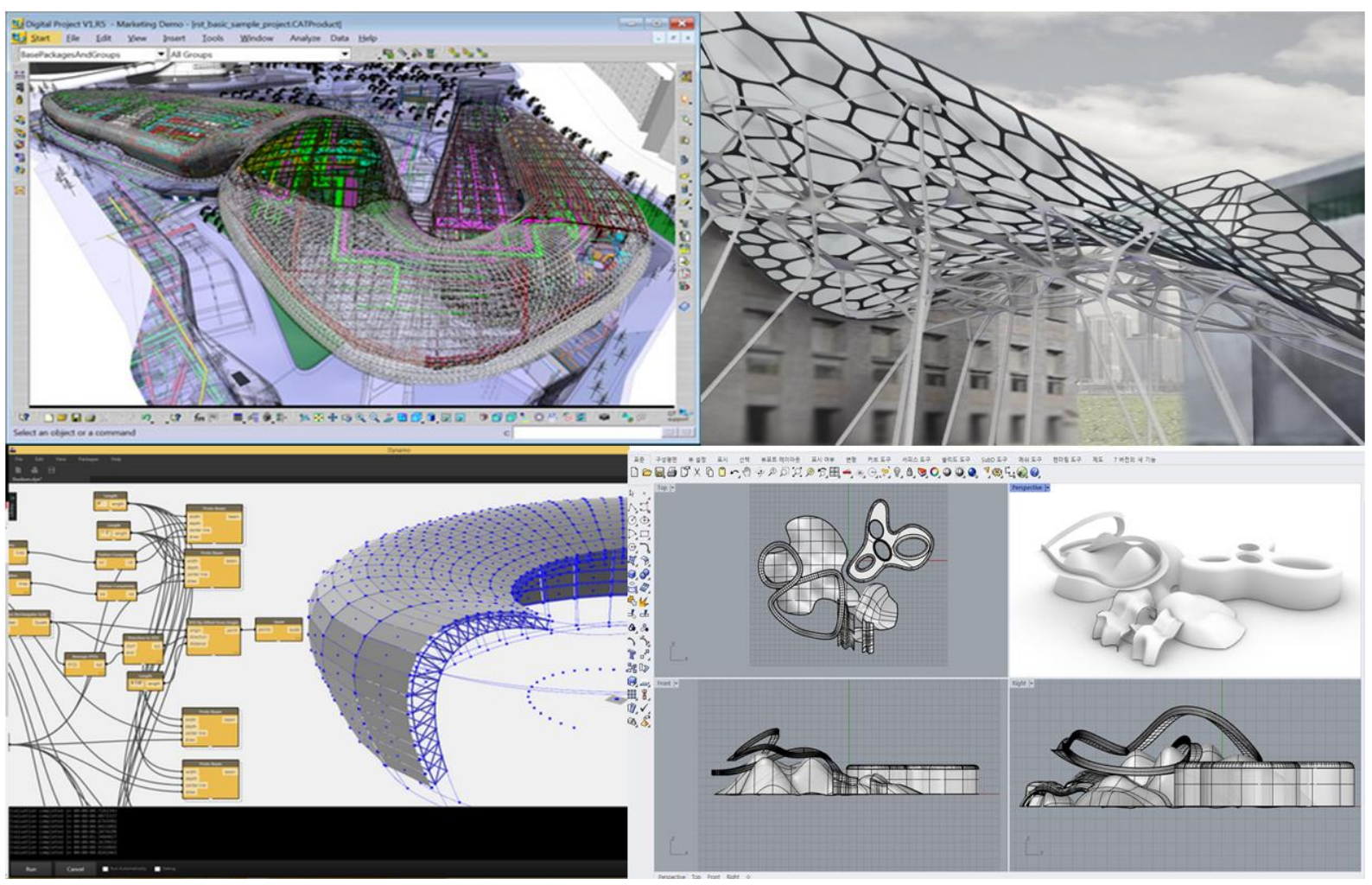

[Fig. 1] Software Supporting Atypical Architectural Design (Digital Project, Grasshopper, Dynamo, Rhino)

Recently, as social demand for atypical architecture has increased, the development and dissemination of tools to support atypical architecture have accelerated. Most of the developed tools support the reproduction of unexpected shapes or support the construction of modeled shapes. [Fig. 1] shows the software that are utilized in atypical architectural design. Digital Project is a tool that supports precise construction, Grasshopper supports generative modeling, and Dynamo supports parametric modeling in conjunction with Revit, a BIM modeling tool. These tools facilitate the design process of architects for atypical structures.

However, designing a space is different from other plastic arts because the user who lives in it is considered. There have been attempts to predict and review user behavior in space, such as statistical or image-based user behavior simulations[9]. Notably, it is more difficult to predict user behavior in atypical architectural spaces, so it is necessary to review the possibilities through human behavior simulation[Fig. 2]. It is necessary to review the habitability of a structure from the outset, and this is more crucial in the design of atypical buildings[Fig. 1] shows the existing atypical design tools, while [Fig. 2] shows the users in completed atypical spaces. A limitation is that it is impossible to simulate the users' behaviors, as shown in [Fig. 2], using only the existing atypical design tools. Furthermore, the interactions between users in the atypical architectural space also need to be simulated. 


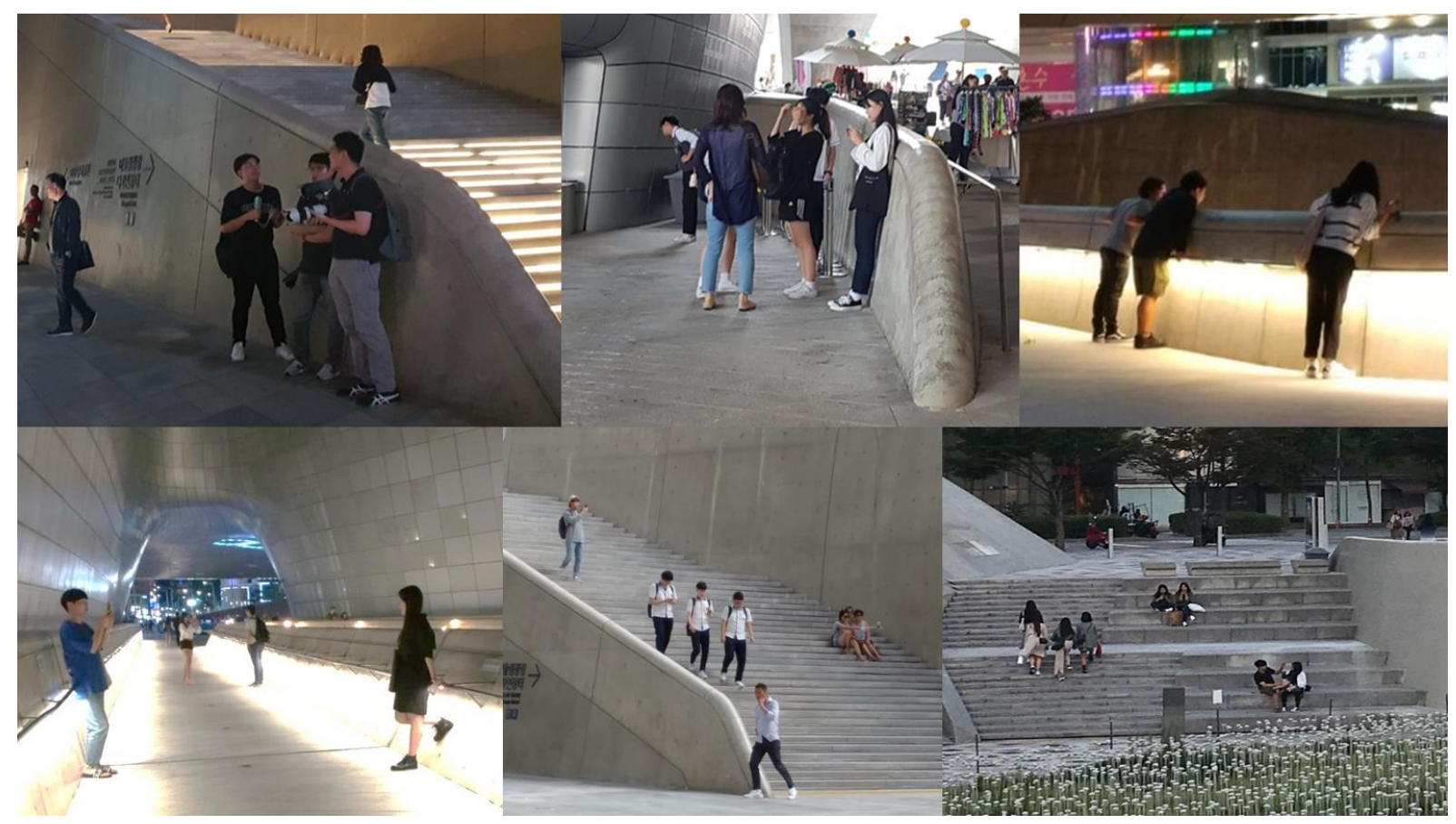

[Fig. 2] Examples of Human Behaviors in Atypical Spaces

\section{Social Interaction for Advanced Human Behavior Simulation}

According to the observations elicited from the field survey, in an atypical building space, the user acts in conjunction with the physical characteristics of the atypical building. In addition, the user interacts naturally with other users in the atypical architectural space. This is somewhat different from a typical space because the basis for interaction is also different. The cause of user interaction in an atypical architectural space could be explained by psychological theory. That is, it can be divided into self-perception, behavioral conformity, in-group bias, and behavior setting, as shown in [Fig. 3]. Each user interaction is initiated by the influence of the atypical architectural space and influences the user's behavior. The user interaction simulation technology in the previously developed atypical architectural space responds to the physical situation of the space. Since this is a simulation that does not take into account all of the social properties of a space, there is a limitation in terms of a more natural simulation[10].

In this study, the concept of dual behavior setting is introduced into the simulation environment. This is because the users' interactions may be greatly affected by the atypical space. An individual's behavior is shaped by the groups to which he/she belongs, but the role of the environment is also emphasized under the assumption that groups are shaped by the environment in which they live. Individuals and groups to which users belong exist within the physical environment in which they are located, and that place ultimately influences the dynamics and outcomes of the group. A built environment is a physical property that can act according to a behavioral setting. This is because people's behaviors in such a space is more influenced by the characteristics of the space than their personal characteristics. Ecopsychologist Roger Barker concluded that most behaviors are determined by the environment in which they occur. Behavioral situations unfold in specific spaces where group members interact. Barker used the term "synomorphy" to describe the correspondence between people and situations. High synomorphy allows people to adapt well to their physical environment and to utilize the components of the environment appropriately. Therefore, the atypical physical environment affects the social behavior of users[11][12]. Simulating social interactions linked with physical 
properties makes it possible to predict user behavior in an atypical space more closely.

Field Survey >> Categorized

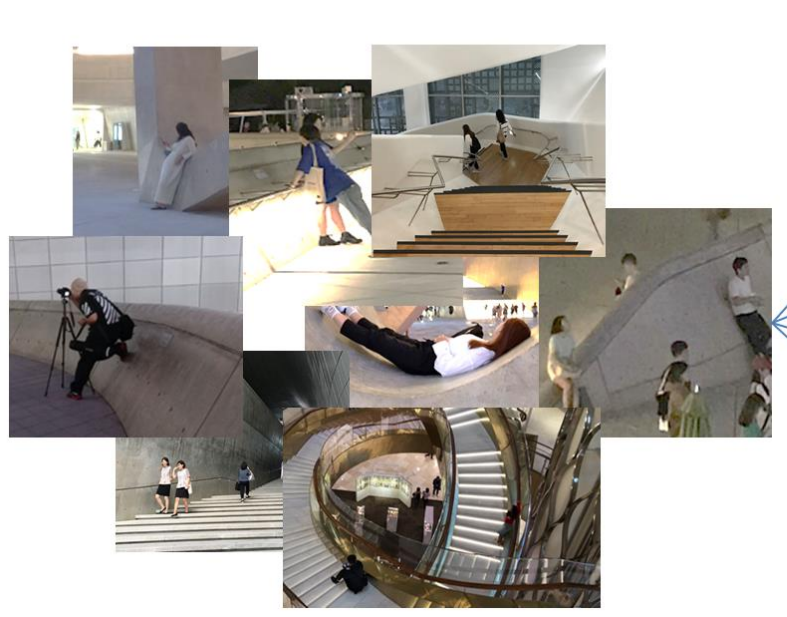

Extracted Patterns

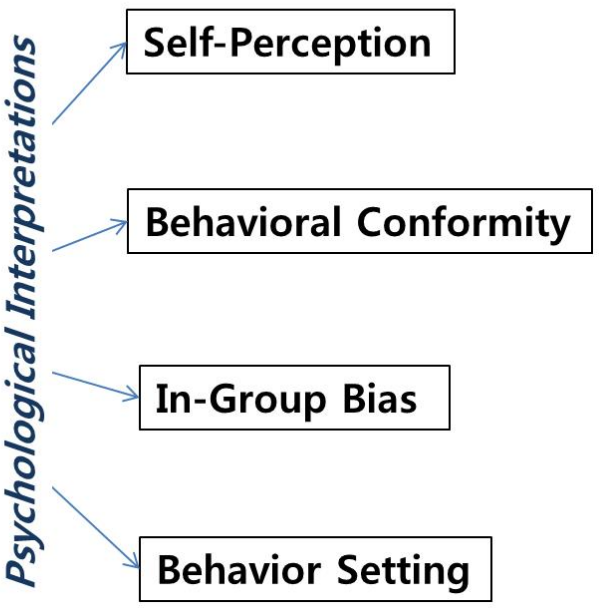

[Fig. 3] Psychological Interpretation and Pattern Extraction of Social Interaction obtained from a Field Survey

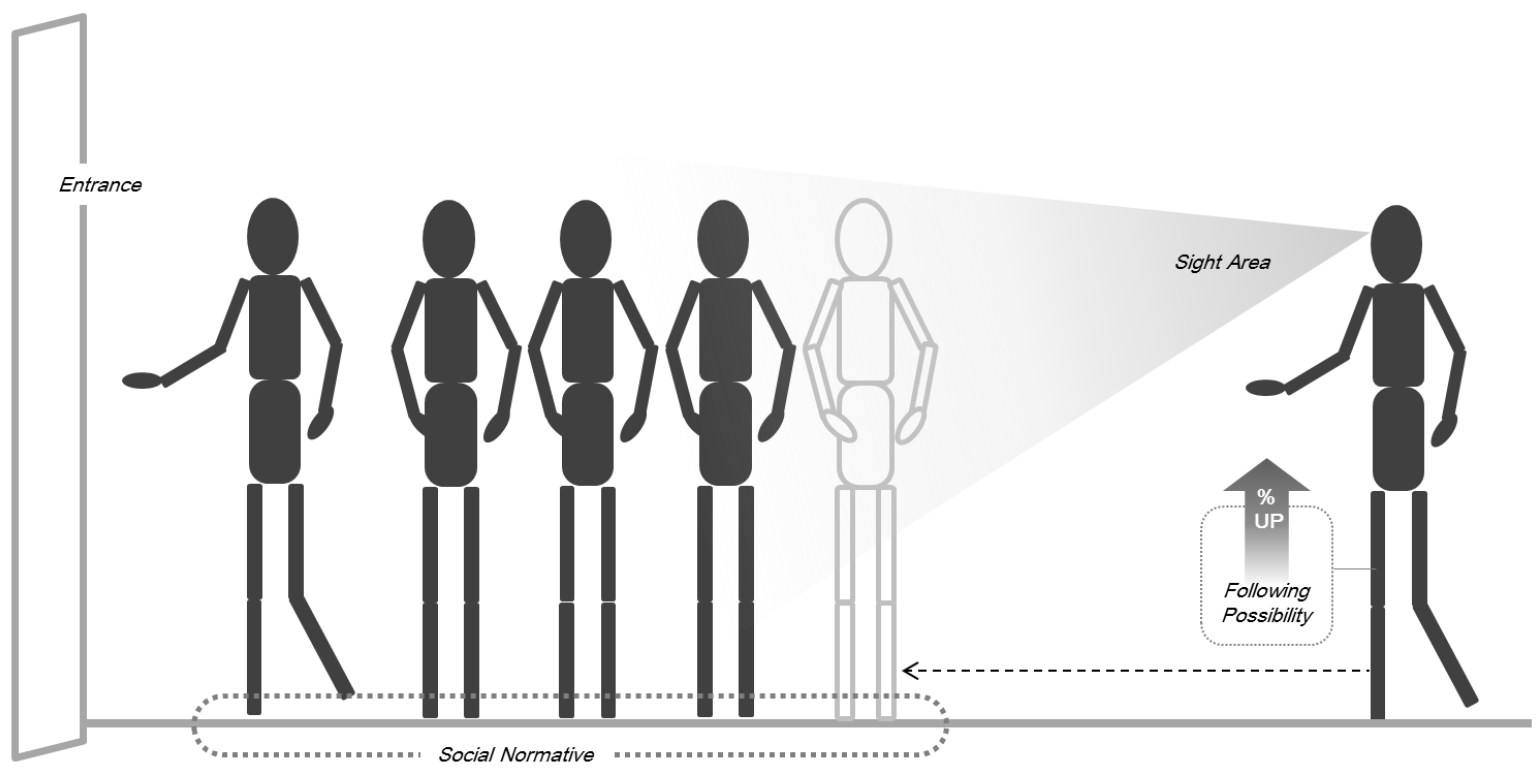

[Fig. 4] Example of Behavior Setting through Social Normative Expressed in the Characteristics of Physical Space[10]

[Fig. 4] and [Fig. 5] show examples of behavior setting in an architectural space[10]. [Fig. 4] shows the social action that conforms to the social normative expressed in the characteristics of the physical space. For example, people naturally line up to take a position, a kind of social consensus that can be interpreted as behavior setting which achieves a social agreement with a physical situation and is generally reproduced. In an atypical space, it is highly likely to be reproduced by adding a physical situation. [Fig. 5] shows the social interaction caused by the surrounding social relationships and situations. As shown in the figure, when there are many people around, it is common for parents to develop a protective instinct toward their children and to move closer to them. When such a situation occurs in an atypical architectural space, it is highly likely to be reproduced in a form that provides the 
cause of the basic behavior of the atypical architectural space and adds social behavior according to social relationships.
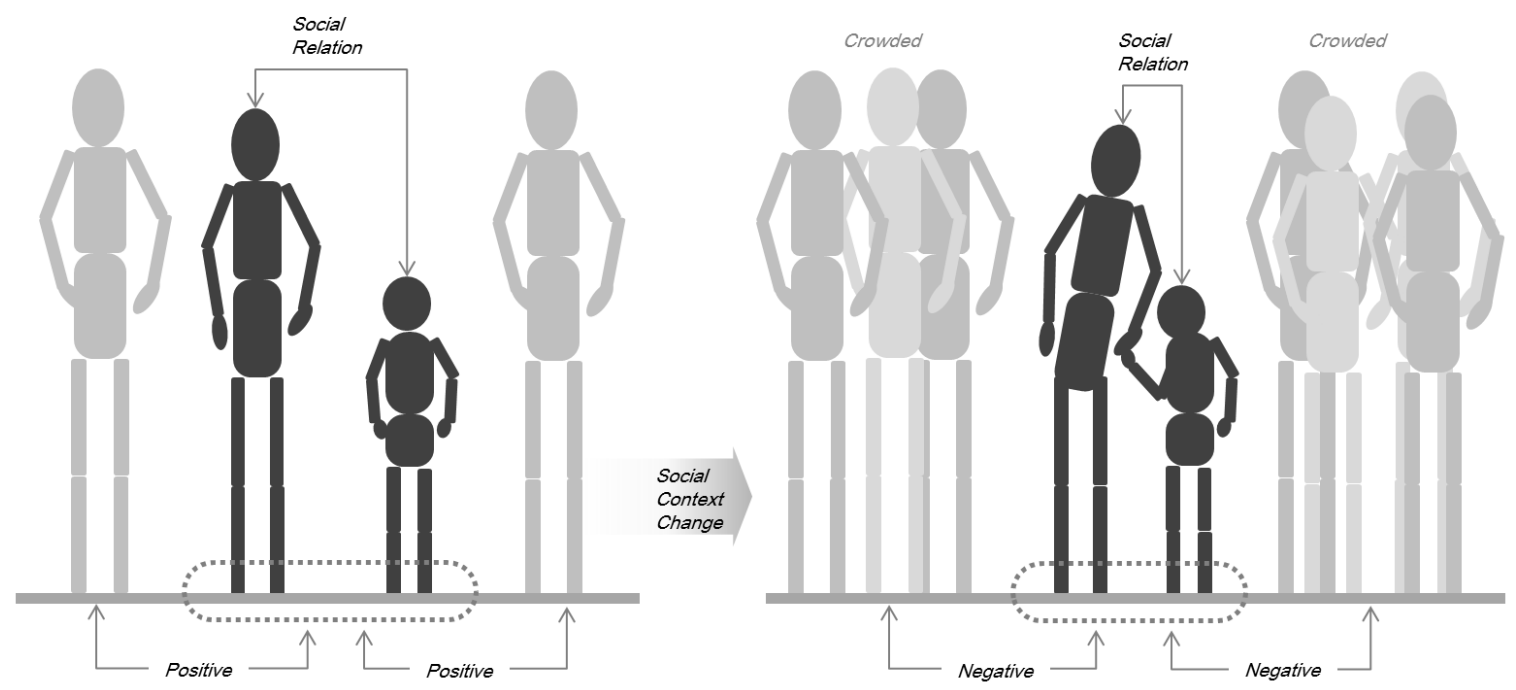

[Fig. 5] Example of Behavior Setting caused by Surrounding Social Relations[10]

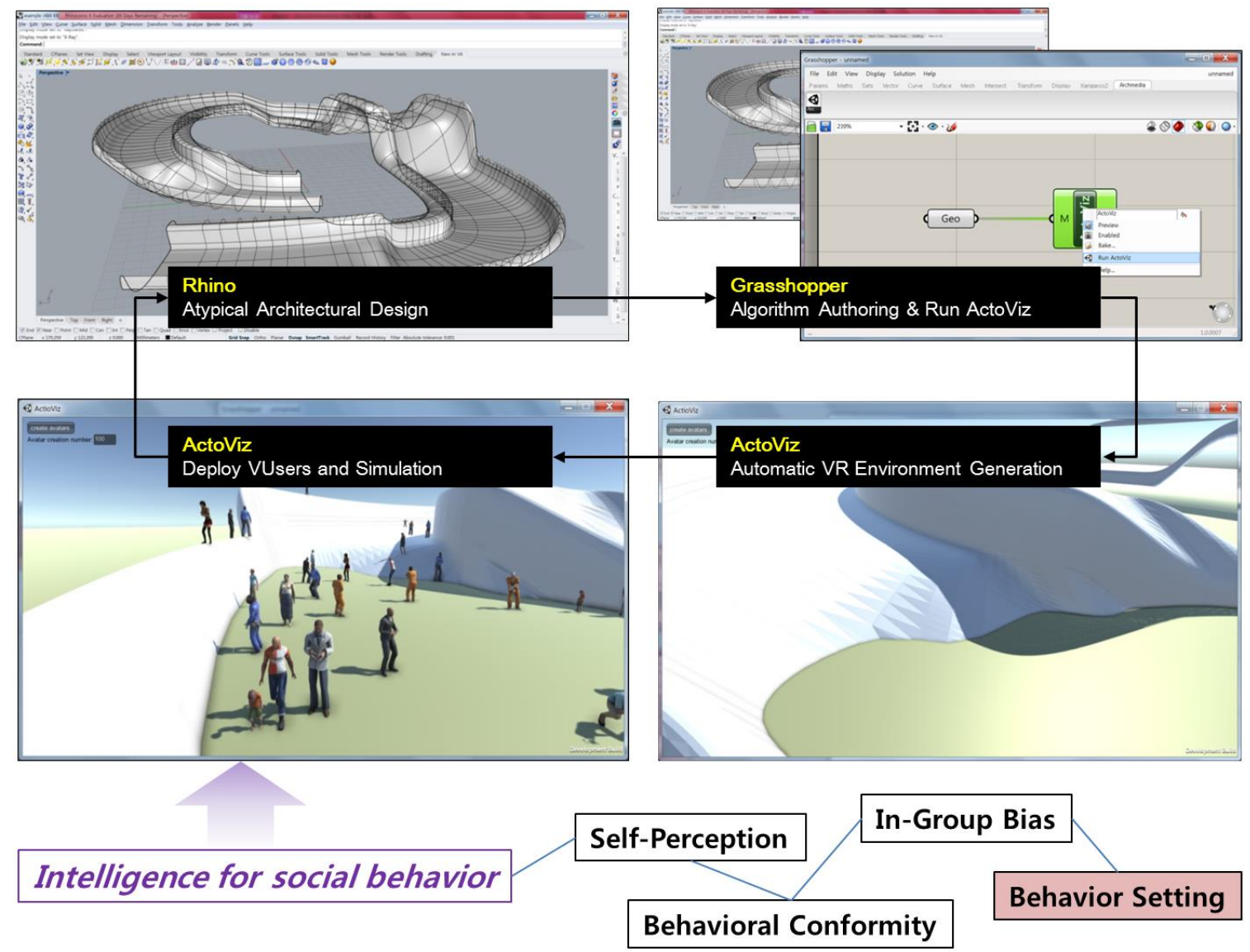

[Fig. 6] Advancement of ActoViz through the Introduction of Intelligent Social Behavior

\section{Developing a Technology to Realize the Behavioral Setting Phenomenon in ActoViz}

In this study, the behavior setting concept was integrated into the previously developed ActoViz 
system. ActoViz is an expert system that supports architects to perform human behavior simulation in the process of atypical architectural design. ActoViz was developed based on Rhino and Grasshopper, atypical architectural design tools. ActoViz reproduces the designed atypical architectural design in real-time as a 3D virtual environment and enables human-shaped agents equipped with artificial intelligence to act freely. [Fig. 6] shows the goal of this study. Ultimately, the objective was to develop an intelligent agent technology that reproduces the social interactions of self-perception, behavioral conformity, in-group bias, and behavior setting in the agent technology that responds to the existing atypical physical situation. This study was conducted mainly for the development of a technology related to behavior setting. [Fig. 6] to [Fig. 8] show the behavior setting concept reproduced based on ActoViz. Since behavior setting has the potential to be reproduced in various ways depending on the physical space situation and the surrounding social situation, it was developed separately for each situation. Thereafter, the applicability of the behavior setting concept was confirmed through several situations. [Fig. 6] shows the appearance of social interaction reproduced through social consensus expressed in the characteristics of physical space. For example, the interaction shown in [Fig. 7] can be reproduced on the inclined floor surface of the atypical space because assistance for the weak people who are walking is needed.

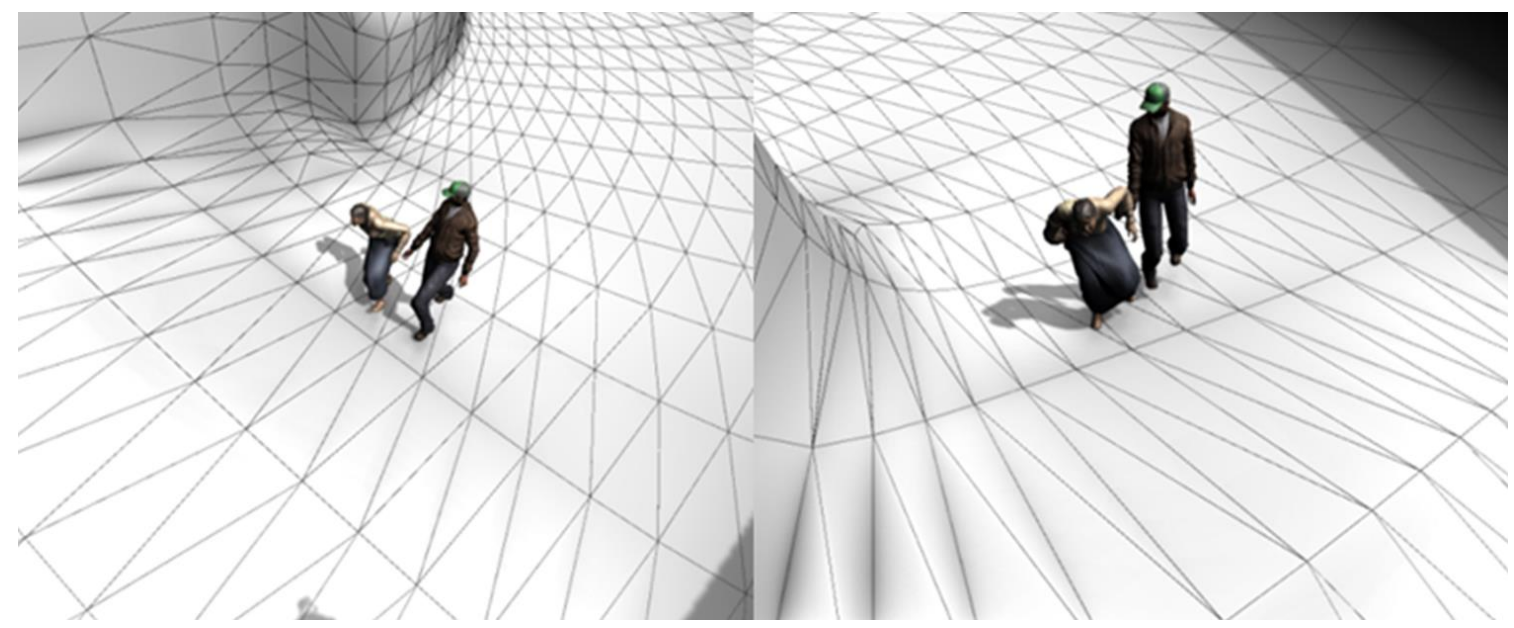

[Fig. 7] Reproduction of Behavior Setting through Social Normative Expressed in the Characteristics of Physical Space

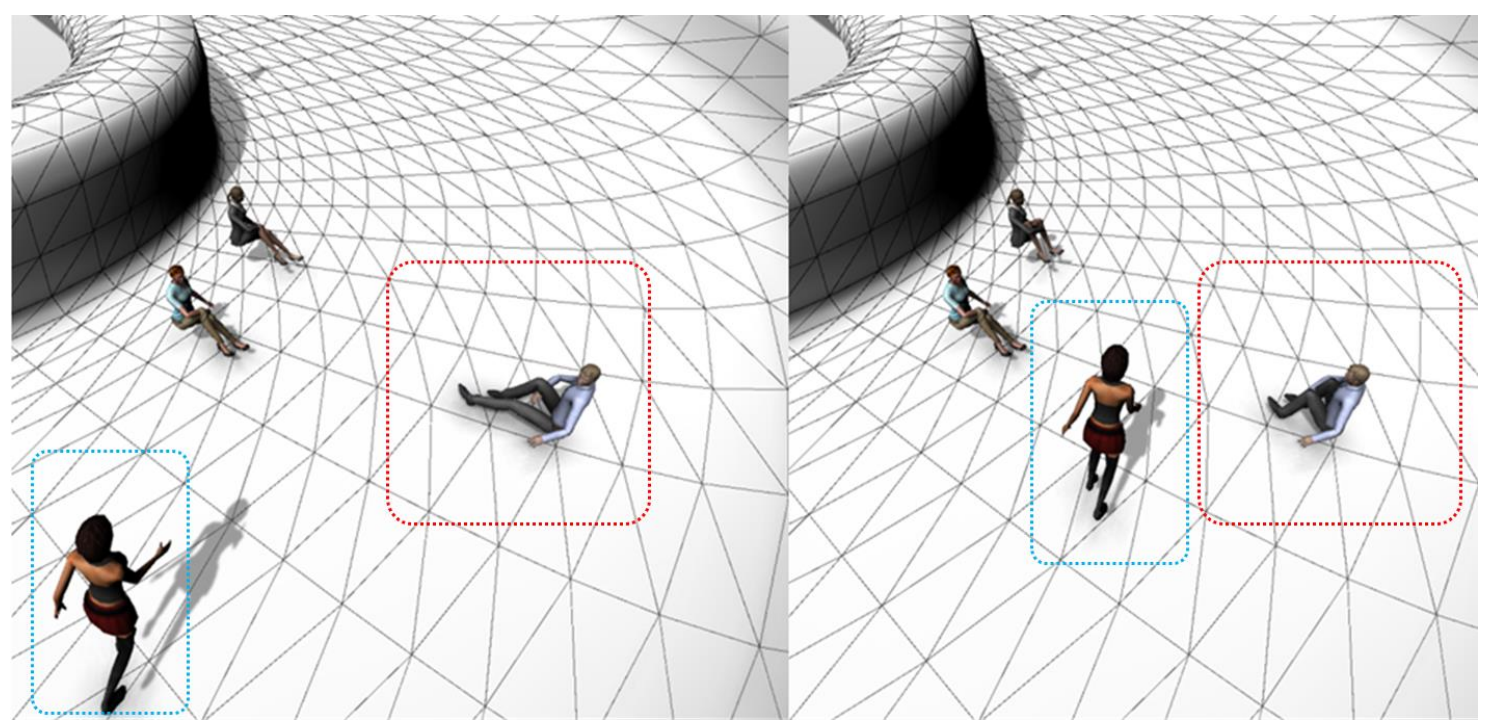

[Fig. 8] Reproduction of Behavior Setting due to Surrounding Social Relations (1) 
[Fig. 8] shows the reproduction of behavior setting due to the social relationships around it. In detail, it shows how to avoid a woman passing in the middle of people gathered in an atypical space by folding the legs slightly to accommodate her. Thus, it can be said that the spatial situation of an atypical space affects social interaction. [Fig. 8] also shows the reproduction of behavior setting due to the social relationships around it. In order not to disturb the group that is performing a specific action in a specific location in an atypical space, it is shown to be moving around in a distant direction.

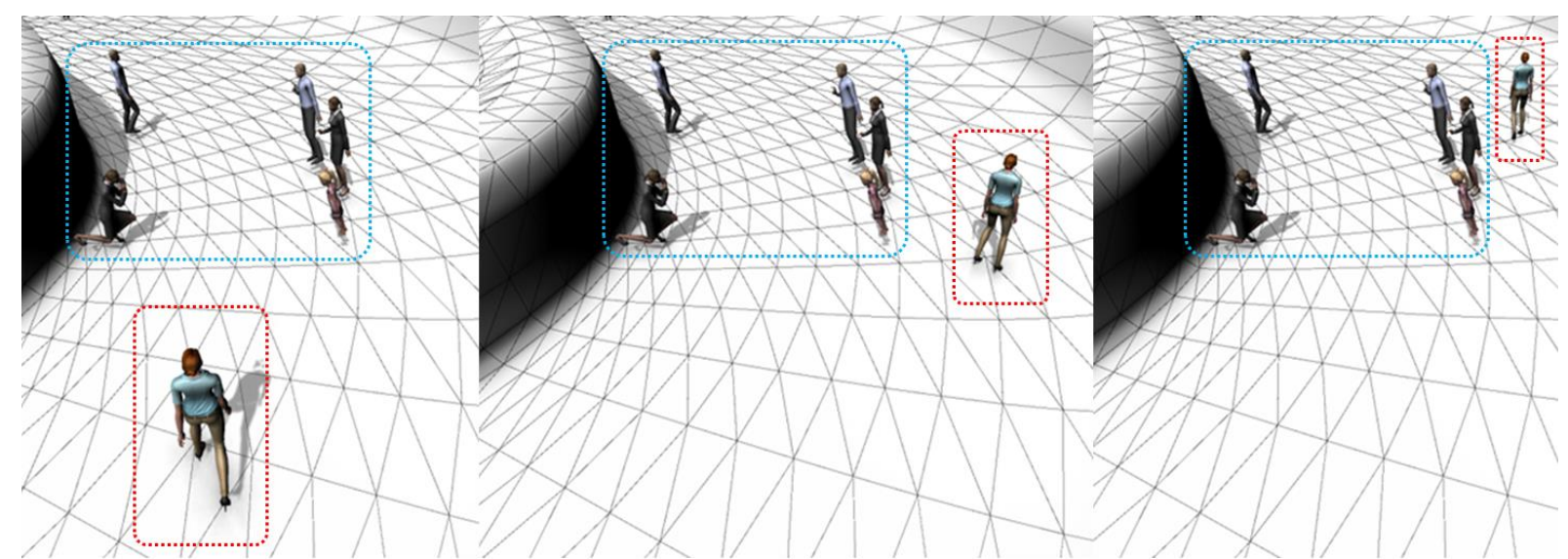

[Fig. 9] Reproduction of Behavior Setting due to Surrounding Social Relations (2)

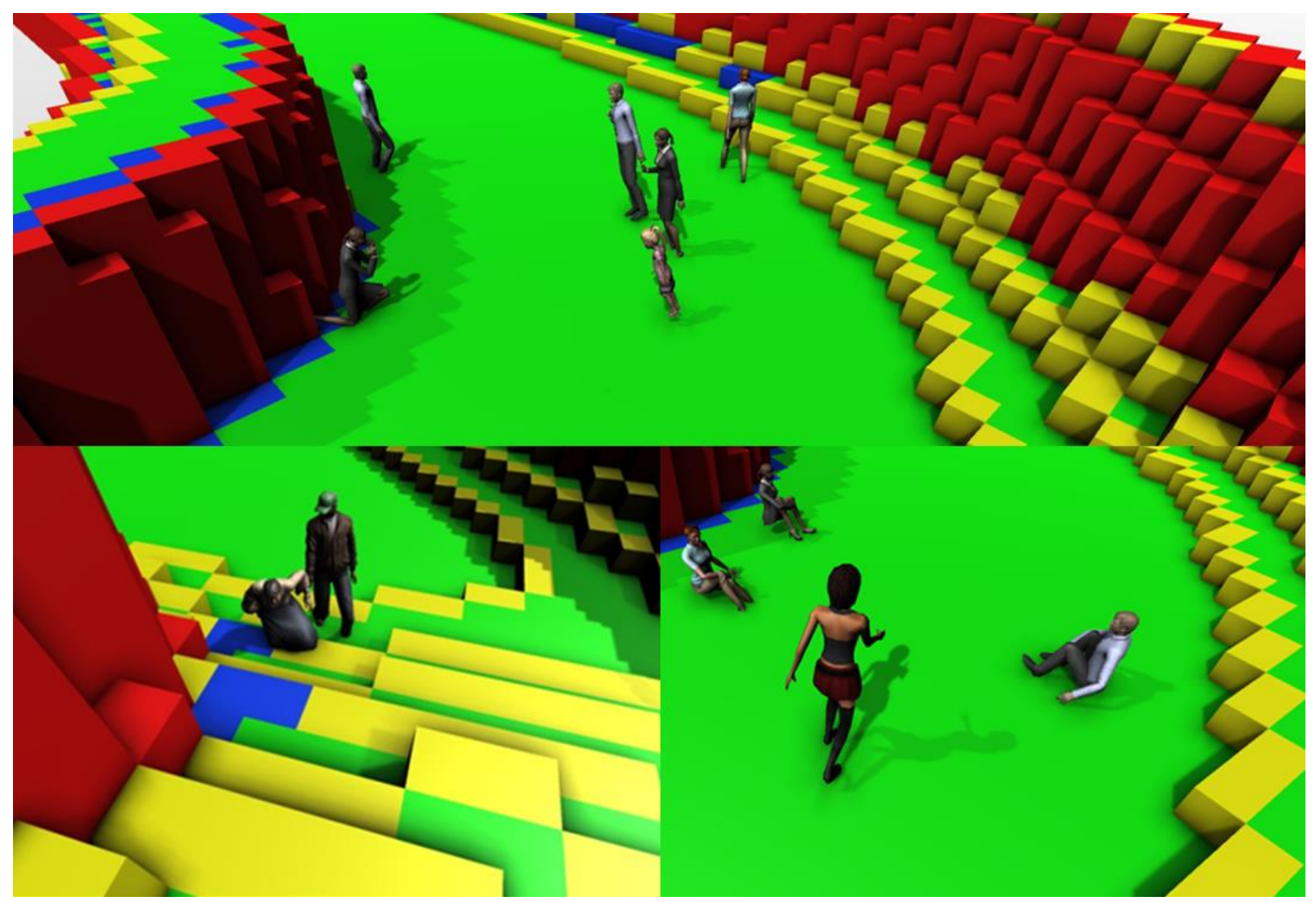

[Fig. 10] Deriving the Location of Social Interaction through Voxelization of Atypical Geometry

The above prototype made it possible to explore the possibility of upgrading the user behavior simulation through the reproduction of behavior setting. In cases like the one shown in [Fig 7], if there is no simulation function for social interaction, the design proceeds simply by anticipating the difficulty of moving the ramp, so there may be no consideration of sufficient space and conditions for 
two or more people to move while helping. In addition, in cases like the one shown in [Fig. 9], if a specific event such as taking a photo occurs and predictions of the user's cluster and the user's behavior are not considered, the space will be designed without understanding the density and behavioral characteristics of the users utilizing the space. [Fig. 10] shows how to derive the location of social interaction through voxelization of atypical geometry. Agents can recognize possible actioninducing points according to the adjacency of the voxels. When the actions of the pre-stored agents start, social actions are developed based on them.

\section{Conclusions}

This paper reports a study on a technological development to advance user simulation for atypical space design. The purpose of this study is to examine the possibility of related technologies through the development of prototype technology, and there is a limit to statistical generalization. In this study, The researchers focused on the agent's technology that reproduces social interaction related to behavior setting. Through the development of some prototypes, it was confirmed that the reproduction of the behavior setting concept can be expressed in a more advanced user simulation. This is because the human behavior reproduced based on Behavior Setting in ActoViz was shown more naturally. Nevertheless, some limitations or areas requiring further development were discovered. First, the behavior setting concept is highly likely to be reproduced in various ways depending on the physical situation of the atypical space and the user's situation. Further research on systematization and generalization is, therefore, warranted to realize this in simulation technology. Second, it is necessary to advance the agent technology that reproduces social consensus. This is a difficult technical problem that eventually needs to be addressed using artificial intelligence technologies, such as deep learning. Third, integration and differentiation with other social interaction skills are required to enable more advanced user simulation by integrating self-perception, behavioral conformity, and in-group bias technologies. Moreover, it is crucial to examine the detailed influence relationship by distinguishing it from other social interactions. Fourth, studying the influence of social interaction technology on the atypical design process should be conducted. It is necessary to optimize social interaction skills by using this system in the design process to check the existence of positive or negative effects on design work.

\section{Acknowledgments}

This work was supported by the National Research Foundation of Korea (NRF) grant funded by the Korean government (MSIT) (NRF-2018R1A2B6005827).

\section{References}

[1] Yehuda E. Kalay, Evaluating and Predicting Design Performance (Principles of Computer-Aided Design), (1st Edition), New York: Wiley Interscience, (1992), pp.329-346.

[2] Y. G. Lee, ActoViz: A Human Behavior Simulator for the Evaluation of the Dwelling Performance of an Atypical Architectural Space, Communications in Computer and Information Science, (2019), Vol.1034, No.1, pp.361-365, DOI:10.1007/978-3-030-23525-3_48

[3] S. W. Hong, Y. G. Lee, Behavioural responsiveness of virtual users for students' creative problem-finding in architectural design, Architectural Science Review, (2019), Vol.62, No.3, pp.238-247, https://doi.org/10.1080/00038628.2019.1594673 
[4] Y. L. Lee, Y. G. Lee, Psychological Interpretation of Human Social Behaviors in the Atypical Architectural Shape, Advances in Intelligent Systems and Computing, (2021), Vol.1269, No.1, pp.33-38, DOI:10.1007/978-3-030-58282$1 \_6$

[5] J. Wagner, Images of Information: Still Photography in the Social Sciences (SAGE Focus Editions), Sage Publications, (1979), pp.217-230.

[6] B. Hiller, J. Hanson, The Social Logic of Space, (Reprint edition), Cambridge University Press, (1989)

[7] J. J. Gibson, The theory of affordances, (From: The Ecological Approach to Visual Perception), (1st Edition), Psychology Press, (2014)

[8] D. A. Norman, Affordances, Conventions and Design. Interactions, ACM Press, (1999), Vol.6, No.3, pp.38-43, https://doi.org/10.1145/301153.301168

[9] J. Archea, The Place of Architectural Factors in Behavioral Theories of Privacy, Journal of Social Issues, (1977), Vol.33, No.3, pp.116-137, https://doi.org/10.1111/j.1540-4560.1977.tb01886.x

[10] Y. L. Lee, Y. G. Lee, A Study on the Strategies of Developing the Autonomous Human Social Behavior of Virtual Users Based on Psychological Interpretation in an Aypical Architectural Shape, Asia-pacific Journal of Convergent Research Interchange, (2020), Vol. 6, No.1, pp.193-202.

[11] D. Myers, J. Twenge, Social Psychology, (13th Edition), McGraw Hill Higher Education, (2019)

[12] Donelson R. Forsyth, Group dynamics, Cengage Learning, (2018) 